Revue des sciences de l'éducation

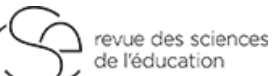

\title{
L’expérience scolaire des jeunes issus de l'immigration maghrébine en France
}

The educational experience of young people with North African

backgrounds in France. Ethnicity and intra-categorical diversity

La experiencia escolar de jóvenes provenientes de la inmigración magrebí en Francia. Etnicidad y diversidad intra-categoría

\section{Abdelhak Qribi}

Volume 42, numéro 2, 2016

Texte reçu le : 21 septembre 2013

Version finale reçue le : 2 novembre 2015

Accepté le : 14 janvier 2016

URI : https://id.erudit.org/iderudit/1038462ar

DOI : https://doi.org/10.7202/1038462ar

Aller au sommaire du numéro

\section{Éditeur(s)}

Revue des sciences de l'éducation

ISSN

1705-0065 (numérique)

Découvrir la revue

Citer cet article

Qribi, A. (2016). L'expérience scolaire des jeunes issus de l'immigration maghrébine en France. Revue des sciences de l'éducation, 42(2), 69-94. https://doi.org/10.7202/1038462ar
Résumé de l'article

La présente étude vise à examiner quelques aspects subjectifs et identitaires de l'expérience scolaire des jeunes issus de l'immigration maghrébine en France. Une enquête par questionnaire réalisée auprès de 120 jeunes des deux sexes, âgés de 18 à 25 ans, issus d'un milieu populaire, permet la mise à l'épreuve de l'hypothèse de la diversité intra-catégorielle. L'analyse statistique, notamment au moyen du $\chi^{2}$, aboutit à la mise en relief de corrélations significatives entre différentes variables constitutives de tissus d'expériences vécues sous le signe de l'aliénation ou de la réalisation personnelle. 


\title{
L'expérience scolaire des jeunes issus de l'immigration maghrébine en France Ethnicité et diversité intra-catégorielle
}

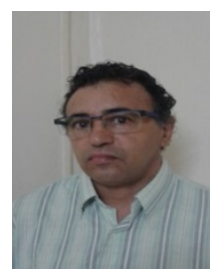

\author{
Abdelhak Qribi \\ Maître de conférences \\ Université de Guyane
}

RÉSUMÉ - La présente étude vise à examiner quelques aspects subjectifs et identitaires de l'expérience scolaire des jeunes issus de l'immigration maghrébine en France. Une enquête par questionnaire réalisée auprès de 120 jeunes des deux sexes, âgés de 18 à 25 ans, issus d'un milieu populaire, permet la mise à l'épreuve de l'hypothèse de la diversité intra-catégorielle. L'analyse statistique, notamment au moyen du $\chi^{2}$, aboutit à la mise en relief de corrélations significatives entre différentes variables constitutives de tissus d'expériences vécues sous le signe de l'aliénation ou de la réalisation personnelle.

MOTS-CLÉS - Expérience scolaire, immigration maghrébine, diversité intracatégorielle, ethnicité, construction identitaire.

\section{Introduction et problématique}

Les représentations collectives et les catégorisations sociales ont la vie longue. En ce qui concerne les conditions d'évolution des jeunes issus de l'immigration maghrébine en France et de leur devenir scolaire et socioprofessionnel, l'approche dominante demeure encore aujourd'hui largement imprégnée par des idées généralistes et globalisantes, comme s'il s'agissait d'un ensemble homogène portant des caractéristiques transposables à chaque individu devenu, en l'occurrence, clone de son groupe d'appartenance. Or, si l'on s'en tient au seul champ scolaire, les études attentives à la diversité des parcours et des expériences commencent à connaître, du moins en milieu spécialisé, une diffusion significative (Brinbaum, 2005; Lorcerie, 2004; Qribi, 2012; Vallet et Caille, 1996).

Nous situant dans ces perspectives qui visent l'appréhension de la singularité de l'expérience scolaire des jeunes issus de l'immigration maghrébine, nous nous proposons de présenter un certain nombre de résultats quantitatifs à partir d'une enquête de terrain portant sur 120 jeunes filles et jeunes hommes âgés de 18-25 ans ayant répondu à un questionnaire de

\footnotetext{
* Mes remerciements vont à tous les jeunes qui ont accepté de livrer une partie d'eux-mêmes en se prêtant à l'exercice du questionnaire et de l'entretien. Ils vont aussi à monsieur Yves Prêteur, professeur de psychologie de l'éducation à l'université de Toulouse le Mirail, pour sa direction de mes travaux de thèse dont est issu le présent travail. Enfin, j'exprime ma gratitude au comité de rédaction et aux lecteurs-arbitres qui, par leurs critiques exigeantes, ont permis l'amélioration de cet article.
} 
72 items sur l'identité et sur l'expérience scolaire et familiale. Le recul acquis par rapport au vécu éducatif et par rapport aux turbulences de l'adolescence, le retour réflexif sur les événements qui ont ponctué un parcours, ainsi que le point de vue exprimé par rapport à ces réalités, représentent des dimensions déterminantes de la construction de l'expérience.

Nous allons nous intéresser, ici, exclusivement à l'expérience scolaire des jeunes dans la mesure où elle représente un champ d'étude spécifique. En effet, bien qu'entretenant des relations étroites avec les conditions sociales d'existence, les ressources et les mobilisations familiales (Lahire, 1995), elle n'en présente pas moins une relative autonomie digne d'intérêt. La massification de l'enseignement secondaire n'a pas engendré uniquement une diversification des parcours scolaires en rapport avec les classes sociales et la hiérarchie du système d'éducation et d'orientation; elle a également produit des effets majeurs sur la construction identitaire des adolescents. Des études sur le public dit nouveaux lycéens issus des milieux sociaux modestes mettent en relief des modes d'élaboration d'expériences scolaires variables selon les positions occupées dans la hiérarchie du système éducatif (Dubet et Martuccelli, 1996). Parmi les figures retenues, on peut mentionner l'opposition entre une véritable subjectivation lycéenne qui passe par l'instauration d'une continuité culturelle entre vie personnelle et projets scolaires d'un côté, et l'aliénation lycéenne que connaissent des jeunes qui ne parviennent pas à se percevoir comme sujets de leurs expériences, de l'autre. D'autres chercheurs se sont intéressés non pas à la gestion de la tension entre logiques scolaires et logiques de vie personnelle référées à une culture juvénile, mais aux rapports au savoir du point de vue du sens et de la valeur qu'accorde un sujet aux processus et produits du savoir (Charlot, Bautier et Rochex, 1992). Il s'agit de processus langagiers, épistémiques et identitaires à l'origine de la mobilisation et de la réussite scolaire. L'étude s'intéresse, là aussi, à l'hétérogénéité des parcours et situations scolaires de jeunes élèves scolarisés en Zones d'éducation prioritaires - Z.E.P. Des constellations différentes y sont mises en lumière autour de la question du sens que les élèves accordent à leur scolarité, et des figures idéales typiques quant aux rapports épistémiques aux savoirs y sont présentées. 
Notre étude s'intéresse au rapport identitaire à l'institution scolaire et à la perception subjective du parcours scolaire et du vécu relationnel. Elle vise à approfondir l'appréhension de la diversité intracatégorielle en se centrant sur un public précis, celui des jeunes issus de l'immigration maghrébine. Quelles sont les caractéristiques les plus saillantes de leur expérience scolaire? Compte tenu de la spécificité ethnoculturelle de cette population, peut-on en cerner les effets sur la construction subjective de ladite expérience? Quels enseignements en tirer sur le plan de la construction identitaire des jeunes?

\section{Contexte théorique}

La corrélation entre appartenances socioculturelles ou ethniques d'une part, et (non) réussite scolaire et socioprofessionnelle d'autre part, a suscité de multiples interrogations et a donné lieu à plusieurs tentatives d'explications. En ce qui concerne les enfants de migrants, le constat d'inégalités scolaires est établi et étayé depuis une trentaine d'années dans un rapport adressé au ministre du travail (Marangé et Lebon, 1982). Dans un autre rapport rédigé par Berque (1985) à la demande du ministre de l'Éducation nationale, les difficultés spécifiques des enfants de migrants sont confirmées. Selon l'auteur, tout se passe comme si la tradition et les idéaux républicains en la matière étaient contrecarrés par de véritables handicaps. Un peu plus de vingt ans après, les experts du Bureau des études statistiques de l'enseignement scolaire soulignent le même phénomène, c'est-à-dire des conditions de scolarisation moins favorables que pour le reste de la population scolaire (Caille, 2007).

\subsection{Des interprétations dominantes de la situation scolaire défavorable des jeunes issus}

\section{de l'immigration}

L'interprétation de ces faits prête à des divergences. Un des clivages que nous retenons concerne l'appréciation du poids de la variable ethnique ou culturelle. Ainsi l'accent a été mis dans un premier temps sur l'apprentissage du français comme condition nécessaire à l'intégration scolaire. Dans un second temps, les recherches ont mis en valeur l'intérêt de l'enseignement des langues et cultures d'origine en lien avec un hypothétique retour dans le pays d'origine, ainsi que sur la promotion d'une pédagogie interculturelle comme facteur enrichissant pour tous les élèves (Abdallah-Pretceille, 1986). Enfin, l'interprétation globale s'est focalisée sur le poids des facteurs socioéconomiques et la problématique de l'intégration des zones d'habitats précaires. Effectivement, en rapport avec le changement des modes d'inscription des populations issues de l'immigration postcoloniale dans la société française dans une perspective de sédentarisation, et l'émergence d'aspirations sociales nouvelles, les 
problématiques scolaires ont évolué et leurs approches politiques et pédagogiques également (Lorcerie, 1999). La création des Zones d'éducation prioritaires s'inscrit dans ce contexte et est en rapport avec ces nouvelles approches basées sur le principe de la discrimination positive et structurant les politiques éducatives de lutte contre les inégalités scolaires (Frandji et Rochex, 2011). Le rapprochement des difficultés scolaires des enfants de migrants et celles des enfants issus de milieux socioéconomiques et culturels dits défavorisés est de plus en plus affirmé. Nous assistons à une relativisation des aspects linguistiques et culturels spécifiques aux enfants de migrants au profit d'une interprétation sociologique globale qui met l'accent sur l'écart commun aux classes populaires par rapport à la norme et à la culture scolaire. Les difficultés scolaires des enfants de migrants seraient l'expression d'une violence symbolique exercée par les classes dominantes au sein de l'école (Bizet, 1985). Le jeune issu d'une minorité ethnique serait en ce sens, selon le sociologue Ben Ayed (2001), une version nouvelle du prolétaire. Dans cette perspective théorique structuraliste, nous retenons le désavantage économique et la distance culturelle variable selon les classes sociales. L'utilisation des statistiques sur la base de la dichotomie français-étranger est critiquée, car, constatent Boulot et Boyzon-Fradet (1988), lorsque la variable socioéconomique est maîtrisée, les parcours et performances des uns et des autres se rapprochent. Dans le même ordre d'idées, une enquête longitudinale portant sur un panel national renforce cette lecture de la scolarité des élèves étrangers ou issus de l'immigration postcoloniale (Vallet et Caille, 1996). Elle permet une relativisation sérieuse de l'échec scolaire chez les enfants de migrants et conclut même à un léger avantage, mais statistiquement très significatif, en faveur du public étudié au moment, notamment, de l'orientation en fin de troisième (élèves de 15 ans). L'hypothèse de la mobilisation des familles autour de la réussite scolaire est privilégiée. Cette dernière idée est confirmée par une autre recherche réalisée par Brinbaum (2005).

\subsection{Critiques, acquis et points d'appui}

Cependant, ces études ne ferment pas pour autant le débat. La construction statistique des résultats peut en effet être interrogée. Il en est ainsi, par exemple, de la validité de l'indicateur de réussite, celui du passage en troisième générale après quatre ans au collège. Mesure-t-on des performances ou des politiques d'orientation des établissements (Lorcerie, 2004)? Les études quantitatives n'escamotent-elles pas les dynamiques internes des établissements et ne négligent-elles pas les processus de fabrication des relations scolaires quotidiennes, laissant ainsi sous silence des différences dignes d'intérêt (Payet, 2000)? D'un autre point de vue, si nous pouvons reconnaître l'aspect positif d'une recherche qui, toutes choses égales par 
ailleurs, met en évidence les origines sociales des inégalités et bat ainsi en brèche une vision essentialiste du jeune issu de l'immigration postcoloniale, nous pouvons aussi rappeler que la population en question se caractérise justement par le cumul des handicaps qui grèvent son existence. Effectivement, comme l'écrit Héran (2002) dans un rapport du Commissariat Général du Plan, ces populations se présentent hic et nunc, toutes choses inégales réunies.

Partant de ces observations critiques et en lien avec nos préoccupations de recherche, nous retenons trois points complémentaires. Le premier réside dans la nécessité de considérer les populations non pas comme des abstractions, mais comme des réalités vivantes. En conséquence, l'égalisation statistique ne doit pas faire oublier que les problèmes réels que vivent les gens ou les acteurs dans un système donné proviennent justement du fait de leur inscription dans des rapports de domination et de discrimination. Ils sont différenciés socialement et ethniquement et, corollairement, l'enjeu social et scientifique réside dans la prise en compte des différences et non dans leur effacement. Le deuxième point réside dans l'intérêt de considérer les travaux sensibles aux différences intracatégorielles encore plus accusées dans le cas des couches défavorisées de la société (Lescarret, 2000) et peut-être encore davantage dans le milieu migrant maghrébin (Chauveau et Rogovas-Chauveau, 1990). Le troisième point réside dans l'intérêt de considérer les dynamiques internes des situations, car c'est du point de vue des interactions au quotidien que les activités d'enseignement et d'apprentissage sont investies et que les identités des élèves et des professionnels se rencontrent et interagissent (Lorcerie, 2004). Ces dynamiques sont aussi tributaires des statuts et des rapports interethniques qui prévalent dans l'environnement social global. Les logiques discriminatoires et ségrégatives infiltrent l'appareil scolaire et dessinent une expérience vécue sur un mode ethnique (Felouzis, Liot et Perroton, 2005; Perroton, 1998, 2000). Toute investigation portant sur l'expérience scolaire des jeunes issus de l'immigration ne pourrait donc ignorer ce dernier mode de différenciation sociale basé sur un sentiment d'appartenance, réel ou imaginaire, instaurant une frontière entre le nous et les autres (Lorcerie, 2004). Un tel sentiment ou une telle croyance ainsi que les effets qu'ils produisent dans la dynamique scolaire ont pu rester longtemps ignorés et marginalisés en sociologie de l'éducation en France, probablement en rapport avec les effets d'une histoire coloniale insuffisamment intégrée (Payet et Van Zanten, 1996a). 


\subsection{Une approche de l'expérience scolaire dans ses dimensions relationnelles et identitaires}

Ce point central étant clarifié, il faut préciser que l'expérience scolaire représente un champ large et recouvre maints aspects de l'activité du sujet. Le concept d'expérience scolaire renvoie à une réalité relative aux multiples interactions des élèves à l'intérieur du système scolaire, aux nombreuses formes d'adaptation mises en œuvre pour se forger une place et acquérir le métier d'élève ainsi que les stratégies mobilisées pour réaliser un parcours donné. L'expérience scolaire renvoie au parcours objectif concret de l'élève, à ses rapports au savoir, mais aussi aux façons dont il a vécu la scolarité à différentes étapes de son parcours scolaire, au sens donné à la fréquentation même de l'école, à la perception de ses rapports avec les enseignants et à l'intérêt éventuel d'un investissement aussi considérable que celui qu'exige l'institution scolaire, durant des années, à la fois de lui-même comme élève et de son milieu familial. Dans ses dimensions subjectives, une telle expérience renvoie à la formation du sujet et au sentiment de se former et de se transformer au contact de l'école et de ses apports non seulement disciplinaires, mais aussi relationnels. Une telle formation et transformation peut s'opérer dans une dynamique positive de réalisation de soi ou une dynamique négative générant un sentiment d'impuissance, d'échec plus ou moins intériorisé, d'aliénation et de non-sens. Retenons toutefois ici l'idée centrale selon laquelle les notions d'intérêt intellectuel, de réalisation de soi et d'ouverture culturelle sont fondamentales et s'avèrent souvent associées à une bonne réussite scolaire. Les élèves qui s'inscrivent dans une telle dynamique parviennent à dépasser la réalisation routinière de tâches morcelées sans véritable projet cognitif ou personnel. Bien au contraire, chez eux, les apprentissages participent au développement et à la transformation de soi (Bautier, Charlot et Rochex, 2000). Qu'en est-il donc au juste des rapports des jeunes à l'institution scolaire et à l'investissement scolaire considéré non pas dans ses dimensions cognitives ou épistémiques, mais plutôt dans ses dimensions identitaires faisant référence à des figures identificatoires et à des projets personnels (Charlot, Bautier, et Rochex, 1992)? Sous cet angle, l'expérience scolaire recouvre des significations et implications qui vont au-delà des savoirs enseignés et appris, pour embrasser l'être dans sa singularité psychique et culturelle, au fondement même de ses interactions, perceptions et projets. En ce qui concerne les lycéens issus de milieux populaires, deux déterminants semblent affecter leur expérience scolaire. D’une part, le rapport à l'apprendre est conditionné par le relationnel noué avec l'enseignant et de ses capacités à faciliter l'entrée dans l'univers du savoir. D'autre part, les interactions avec les pairs ainsi que la dynamique et l'ambiance d'établissement pèsent considérablement dans leur 
construction identitaire et leurs représentations de leur valeur (Périer, 2004). Tout sujet étant actif dans son appropriation du social, la question de la singularité des constructions et des identités nous paraît, en ce sens, fondamentale.

Pour la présente recherche, une hypothèse centrale a guidé notre investigation : en dépit de leur appartenance socioethnique commune, les expériences scolaires des jeunes issus de l'immigration maghrébine se présentent sous le signe de la diversité. Plus précisément, si certains parviennent à construire un parcours dans lequel ils se réalisent personnellement, d'autres, au contraire, vivent des expériences difficiles marquées du sceau de l'échec et du non-sens. Nous formulons par ailleurs l'hypothèse complémentaire de l'existence de corrélations significatives entre les divers aspects de l'expérience scolaire, tant dans ses composantes objectives que dans ses dimensions subjectives.

\section{Méthodologie}

\subsection{Sujets}

Compte tenu des objectifs de l'enquête et de la particularité de l'hypothèse de la recherche, la maitrise de la variable socioéconomique et ethnique a représenté un impératif incontournable. Une relative homogénéité de l'échantillon à ces niveaux socioethniques, mais aussi démographiques et biographiques (du point de vue des conditions globales de socialisation) a été donc recherchée. L'enquête a porté sur 120 jeunes âgés de 18 à 25 ans, des deux sexes : 70 femmes et 50 hommes. Une partie d'entre eux habitent au nord-est de la France, l'autre au sud-ouest. Ils sont majoritairement célibataires (85\%). Ils sont tous issus de familles où les deux parents sont d'origine maghrébine, très majoritairement de milieu ouvrier. En effet, $42,5 \%$ des pères ont un statut d'ouvrier qualifié et $41,7 \%$, celui d'ouvrier non qualifié. Le statut socioprofessionnel des mères qui ont un métier est semblable : $66 \%$ sont femmes au foyer, $16 \%$ ouvrières non qualifiées et $7 \%$ ouvrières qualifiées. Les parents ont un niveau scolaire plutôt faible : $32,5 \%$ des mères et $17 \%$ des pères n'ont jamais été scolarisés; $17 \%$ des mères et $27 \%$ des pères ont connu uniquement l'école coranique. Ils se répartissent ensuite essentiellement entre le niveau primaire $(20 \%$ des pères et $24 \%$ des mères) et secondaire ( $21 \%$ des mères et $27 \%$ des pères). Le niveau universitaire demeure marginal. Les sujets interrogés sont nés en France ( $85 \%$ ) ou y sont venus avant l'âge de 5 ans (15\%) dans le cadre du regroupement familial. L'échantillon ne comporte pas d'enfant unique et les fratries nombreuses prédominent. Sur le plan scolaire, $51 \%$ de sujets ont atteint le niveau supérieur, $43 \%$ le niveau lycée et $6 \%$ le niveau collège. Du point de vue de l'occupation au moment de l'enquête, nous distinguons deux catégories : $51 \%$ sont inscrits en formation 
générale (42\%) ou professionnelle ( $9 \%$ ); et $49 \%$ ne relèvent pas de la formation et sont donc soit sans emploi (24\%), soit occupant un emploi stable (10\%) ou un emploi précaire (15\%).

\subsection{Instrumentation}

Les travaux sur l'expérience scolaire utilisent des méthodologies variées. En effet, alors que certains chercheurs optent pour des méthodologies qualitatives basées sur les bilans de savoir (Charlot, Bautier et Rochex, 1992) ou l'intervention sociologique (Potvin, Carignan, Audet, Bilodeau et Deshaies, 2010), d'autres optent pour une démarche quantitative (Courtinat et De Léonardis, 2010; De Léonardis, Féchant et Prêteur, 2005). Compte tenu des objectifs de la recherche visant l'appréhension de la diversité des expériences scolaires sur un échantillon relativement important, et la mise en relief des liens entre les dimensions constitutives de celles-ci, nous avons opté pour une démarche quantitative. Le questionnaire comme outil de recueil de données paraît adapté pour l'opérationnalisation des hypothèses en termes de dimensions et d'indicateurs. Outre les informations sociobiographiques, 16 items du questionnaire ont servi à appréhender six dimensions de l'expérience scolaire. Les dimensions sont appréhendées à travers une échelle d'attitudes et d'appréciations subjectives, comportant, selon les items, 3 ou 4 niveaux. Une telle échelle permet de nuancer les réponses binaires en introduisant une graduation. La première dimension renvoie au sentiment du jeune quant à sa perception du jugement des enseignants sur ses capacités scolaires comme bon élève, mauvais élève ou élève moyen, aussi bien à l'école primaire, au collège (11-15 ans) qu'au lycée (15-18 ans) . La deuxième dimension renvoie au sentiment éventuel de discrimination raciale ressentie de temps en temps, jamais, fréquemment ou rarement durant les trois mêmes niveaux de scolarité. La troisième dimension concerne les rapports avec les enseignants exprimés dans des termes affectifs, c'est-à-dire selon qu'ils étaient vécus comme plutôt agréables et sympathiques, désagréables et antipathiques ou indifférents durant les trois cycles scolaires. La quatrième dimension renvoie au sens de l'école selon qu'elle a été fréquentée avec plaisir et intérêt, par contrainte et obligation ou par habitude et pour rencontrer les copains. La cinquième dimension a trait à l'appréciation du jeune de l'intérêt de l'école en général et à son niveau de satisfaction (allant du très satisfait jusqu'au pas du tout satisfait, en passant par assez satisfait et assez peu satisfait) par rapport aux apports scolaires tant sur le plan professionnel que personnel. Enfin, la sixième dimension concerne des aspects concrets du déroulement de la scolarité tels que le redoublement éventuel d'une classe (allant de jamais jusqu'à plus de 2 fois en passant par une fois et deux fois) ou encore le jugement global du jeune sur ses résultats scolaires selon qu'il les situe plutôt comme supérieurs à la moyenne, 
justes à la moyenne, au-dessous de la moyenne ou très insuffisants. Le choix de l'ensemble de ces items prend appui sur les enseignements tirés de la recension des écrits de recherche, et notamment des travaux de Charlot, Bautier, Rochex (1992), Dubet et Martuccelli (1996) par rapport à la question du sens de l'expérience scolaire d'une part, et ceux de Payet (2000), Lorcerie (2004) et Perroton (1998, 2000), mettant en relief le poids de la variable ethnique dans ladite expérience, d'autre part.

\subsection{Déroulement}

Recruter 120 jeunes répondant à des critères précis, dans leur milieu naturel de vie, n'est pas chose aisée. C'est donc essentiellement à travers notre réseau personnel, associatif et professionnel que ce travail a pu être effectué. Au moins trois facteurs limitent les biais susceptibles d'affecter ce choix de méthode : le premier réside dans l'hétérogénéité sociale du réseau; le second se manifeste dans la diversité des situations d'occupation des jeunes et des aires géographiques d'habitat mentionnées supra; le troisième est l'absence d'une relation directe avec les personnes interrogées. Nous avons rencontré les jeunes dans leurs lieux de vie habituels (quartiers, maisons de jeunes...). La passation du questionnaire a été réalisée de manière individuelle avec une administration dite indirecte. Chaque passation a duré de 40 à 70 minutes selon les cas. Très coûteux en termes de temps, un tel mode nous a permis un recueil de données de qualité. Les établissements sociaux ont souvent servi de lieu d'accueil pour cette enquête. Le volontariat, la disponibilité et la confiance des sujets ont toujours été recherchés, et, pensons-nous, obtenus.

\subsection{Méthode d'analyse des données}

L'exploitation du questionnaire a été réalisée au moyen du logiciel SPSS. Elle a suivi deux phases et a permis la réalisation des deux objectifs poursuivis : la première phase est celle du tri à plat. Elle a permis de vérifier l'effectivité de la diversité des expériences scolaires. La seconde phase est celle du tri croisé. Elle a permis la mise en relief d'un certain nombre de corrélations à l'intérieur du champ étudié. Quelques regroupements entre modalités de réponses ont été effectués pour rendre l'exercice du $\chi^{2}$ conforme aux règles de la statistique (Lignon, Rigal et Thiong Ly, 2000, p.73). Lorsque les différences observées entre groupes construits nous ont paru assez élevées pour s'expliquer par le seul effet de variables non contrôlées ou le hasard, nous avons rejeté l'hypothèse nulle et considéré autrement l'observation. Le seuil minimum admis a été de 0,05 , soit des valeurs jamais inférieures à 5,99 pour 2 degrés de liberté (ddl) ou 9,48 pour 4 degrés de liberté. Un tel seuil est considéré par 
les statisticiens comme un compromis raisonnable qui évite la rigueur trompeuse qu'offrirait un seuil trop élevé ( $\mathrm{p}<0,0001$, par exemple) ainsi que le non-sens du hasard associé à un seuil trop bas (Delhomme et Meyer, 2002, p.120).

\subsection{Considérations éthiques}

Tous les sujets ayant participé à cette enquête l'ont fait de façon volontaire. Tous ont été informés individuellement, et bien avant l'administration du questionnaire, des thèmes de la recherche, de son caractère universitaire, de son anonymat et du respect absolu de toutes les réponses données, sans aucun jugement de valeur. Les items abordés étant sensibles, la relation de confiance nous semblait répondre à un impératif à la fois éthique et épistémologique. Quand une question semblait incompréhensible, l'enquêteur la précisait, dans une neutralité bienveillante, car l'échange paraissait être une ligne éthique au service même de la rigueur méthodologique.

\section{Résultats de l'enquête}

\subsection{La scolarité au miroir du regard enseignant}

Interrogés au sujet des appréciations que portaient les enseignants sur leur scolarité durant les différents cycles du parcours scolaire, les élèves témoignent dans leurs réponses d'une véritable diversité. La répartition des sujets selon les appréciations positives d'une part, et celles plutôt moyennes d'autre part, est assez équilibrée. Ainsi le nombre d'élèves qui s'est senti considéré comme bon élève oscille entre 43 et $54 \%$ et celui qui s'est senti considéré comme élève moyen atteint 38 à 47,5\% des effectifs. À cela s'ajoute la situation d'un petit noyau constitué de 7 à $9 \%$ qui s'est senti considéré comme plutôt mauvais élève à l'un ou l'autre niveau de la scolarité. De telles observations suscitent au moins deux réflexions : la première concerne l'effectivité d'une véritable diversité des parcours des jeunes. Elle est très prononcée. Toute étude gagnerait à tenir compte de ces différences intracatégorielles. La deuxième réflexion consiste à souligner le caractère préoccupant du nombre élevé de jeunes qui se sentent considérés comme plutôt mauvais élèves ou élèves moyens. Le regard enseignant positif ou négatif sur un élève n'affecte-t-il pas la relation pédagogique et le rapport aux apprentissages? L'intérêt pour la matière ne reste-t-il pas largement déterminé par le rapport à l'enseignant (Dubet et Martuccelli, 1996)? Dans cette même perspective, il est également préoccupant de relever que 7 à $8 \%$ d'élèves pensent avoir connu fréquemment une discrimination raciale ou de temps en temps dans une proportion de 19 à $30 \%$. Une telle réalité signifie que l'école est loin d'être complètement épargnée des tensions sociales, 
culturelles et idéologiques qui traversent la société. Il y a là une violence qui pourrait aider à rendre compte d'un certain désordre scolaire et plus généralement de la défiance qu'exprime une partie de la jeunesse vis-à-vis des institutions sociales, école comprise. Certes, la problématique de l'humiliation des élèves dans le système scolaire français est assez générale (Merle, 2005), mais il est probable qu'elle se confond, dans le cas des jeunes issus de l'immigration maghrébine, avec la question ethnique. C'est du moins ce que soutiennent des travaux évoquant le racisme des professeurs dénoncé par les élèves orientés vers des filières dévalorisées (Zephir, 2007) et le développement dans ce type de contexte de réactions ethniques de fierté et de révolte (Perroton, 2000).

Toujours sur un versant subjectif, la question des liens des jeunes avec leurs enseignants est abordée à travers un autre item, en termes de sympathie, d'antipathie ou d'indifférence. Et l'on constate que si une majorité importante juge ces relations plutôt agréables et sympathiques dans 61 à $67 \%$ de cas selon les différents niveaux scolaires, il reste un noyau comparable à celui repéré sur la question de la discrimination raciale se situant entre 6 et $11 \%$ et qui juge ces relations plutôt désagréables et antipathiques. De même 24 à $30 \%$ des élèves jugent ces rapports aux enseignants comme indifférents. Existe-t-il un effet ethnique conditionnant les relations affectives entre enseignants et élèves? Y aurait-il, dans la classe, un langage non verbal jouant en défaveur des enfants des classes populaires et de ceux issus de l'immigration plus particulièrement (Zimmermann, 1982)? Des stigmates ou des formes infraconscientes de jugements défavorables, fondés sur des critères extérieurs au travail scolaire tels que le style d'habillement et la manière de s'exprimer ne contribuent-ils pas à installer la distance entre l'enseignant et l'élève (Payet et Van Zanten, 1996b)? Les résultats de l'enquête apportent une réponse nuancée. Les sentiments positifs ressentis par les jeunes restent majoritaires. Cependant, compte tenu de la proportion de ceux qui disent avoir ressenti qu'on les considérait de façon négative, l'hypothèse d'un effet de stigmate s'avère tout à fait sérieuse. Certes, il est plausible que certains élèves puissent utiliser l'argument racial pour justifier une disqualification scolaire ou des difficultés d'apprentissage, mais il n'en demeure pas moins vrai que les usages réellement stigmatisants de l'ethnicité opèrent au désavantage du public concerné (Perroton, 2000).

\subsection{Sens de l'école et appréciation de son intérêt}

Cette question nous a permis de relever une diversité de motivations dans les différents stades du parcours scolaire. Ainsi, une majorité de jeunes élèves affirme avoir été à l'école avec plaisir et intérêt, soit $50 \%$ de l'effectif en ce qui concerne l'école primaire, $44 \%$ du collège 
et $62 \%$ du lycée. Cette tendance est plutôt stimulante scolairement. Ceux qui ont fréquenté l'institution scolaire par contrainte et obligation représentent seulement 14 à $15 \%$ et ceux qui s'y rendaient plutôt par habitude et pour rencontrer les copains représentent 35,2 \% au niveau du primaire, $40,6 \%$ celui du collège et 22,5\% au niveau du lycée. Ces deux derniers groupes seront, au cours de l'exploitation statistique, regroupés, par opposition au groupe manifestant plaisir et intérêt scolaire. Notons au passage qu'un tel résultat fournit un nouvel élément en faveur de l'hypothèse de l'hétérogénéité scolaire dans la population étudiée.

Enfin, et toujours en lien avec la question du sens de l'école et de son intérêt, les jeunes enquêtés ont été invités à exprimer une appréciation globale, à effectuer une sorte de bilan individuel par rapport à l'école. Quel est l'intérêt de l'aventure scolaire tant sur le plan personnel que professionnel? Comment les jeunes interrogés jugent-ils les apports de l'investissement scolaire? Les résultats obtenus contrastent de manière forte avec l'image dominante en la matière, insistant sur le discrédit qui frapperait l'école aujourd'hui en lien avec la problématique de l'emploi d'une part, et l'effritement des structures traditionnelles de l'autorité pourvoyeuses de sens, d'autre part. Invités à exprimer leur niveau de satisfaction quant à ce à quoi sert ou servira l'école sur le plan personnel, presque un tiers des personnes interrogées, soit $30 \%$ se dit très satisfait et presque la moitié de la population de l'enquête, soit 49,2 se dit assez satisfaite. Seules des minorités de $15,8 \%$ et de $5 \%$ se disent respectivement peu satisfaites ou pas du tout satisfaites. Ce résultat est, on le verra plus loin, en corrélation avec l'échec scolaire. Cependant, tout bien considéré, il y a lieu de penser que si les institutions traditionnelles de socialisation souffrent d'un quelconque déclin (Cortesero, 2010), il conviendrait de rédiger un chapitre à part sur les liens de ces jeunes à l'école en rapport avec leur histoire familiale et leur subjectivité; et on apprécierait peut-être mieux la place particulière de cette institution dans leur parcours personnel et social. Les niveaux de satisfaction exprimés quant à l'intérêt professionnel de l'école restent également assez élevés. $23,3 \%$ des jeunes interrogés affirment être très satisfaits et 45,8\% assez satisfaits contre $12,5 \%$ de jeunes pas du tout satisfaits et 18,3\% peu satisfaits. De tels résultats sont d'autant plus importants qu'ils contrastent avec la réalité effective des personnes concernées sur le marché de l'emploi, réalité marquée par la précarité, la discrimination et l'incertitude (Benguigui, 2006; Glaude et Borrel, 2002). On pourrait se demander si les jeunes n'expriment pas à travers ce positionnement une conscience autrement plus aigüe des enjeux de l'insertion professionnelle, ce qui les conduit à sérier autrement les mécanismes en jeu et à percevoir plus adéquatement les causes qu'en se focalisant sur l'école comme source des problèmes rencontrés. 


\subsection{Aspects du déroulement scolaire concret}

Il s'agit ici, non pas d'un examen exhaustif et objectif de la scolarité des enquêtés à travers une confrontation de leur propre appréciation avec les résultats effectivement obtenus, mais uniquement d'une approche de leur sentiment en la matière sur une base déclarative. Nous ne visons pas la mesure véritable des notes et des moyennes obtenues à telle ou telle étape du parcours, mais l'appréhension d'une vérité subjective qui, même si elle ne reflète pas les faits exacts d'un déroulement scolaire, donne toutefois un aperçu sur ce qu'a été une scolarité du point de vue du sujet.

Invités à porter un jugement global sur leurs performances scolaires, les jeunes interrogés expriment des appréciations très variables. En effet, nous retrouvons deux groupes parfaitement équilibrés constitués de $45 \%$ qui estiment avoir eu des résultats supérieurs à la moyenne et $45 \%$ qui estiment avoir eu des résultats juste à la moyenne. Une minorité de $10 \%$ juge ses résultats inférieurs à la moyenne. Ces appréciations recoupent dans une très large mesure celles déjà rapportées et qui concernent la perception, par le jeune, de l'appréciation des enseignants.

L'examen du redoublement éventuel au cours de la scolarité représente l'autre volet de la question. Il en est le corollaire. Il sanctionne les différences d'acquisitions attendues selon un rythme et un calendrier donné. Le nombre d'élèves n'ayant jamais redoublé atteint seulement $25,6 \%$, celui ayant redoublé une fois représente $40,8 \%$ et celui qui a redoublé deux fois représente $25,8 \%$. À cela s'ajoute le cas d'un petit noyau de 7,5\% qui enregistre plus de deux redoublements. Ces données concernent l'ensemble du parcours scolaire depuis le primaire jusqu'à l'Université. Ce dernier cycle constitue, notamment dans la première année, un véritable obstacle. L'accompagnement scolaire en milieu populaire est loin d'être systématique. Seulement $28,8 \%$ des jeunes interrogés affirment avoir bénéficié d'une aide au sein de leur famille au cours de leur scolarité, et $15,3 \%$ dans le cadre associatif ou des cours privés. 55,9\% affirment avoir travaillé seuls. Or, la préparation scolaire familiale et environnementale est décisive en matière de réussite scolaire (Feyfant, 2011; Lahire, 1995); les espaces intermédiaires aux côtés des familles et de l'école, aussi (Glasman, 2001). Cependant, si l'analyse du redoublement témoigne, dans les écrits de recherche spécialisés, des inégalités d'éducation selon les milieux sociaux, et si le redoublement au primaire notamment représente un prédicteur de ce que pourrait être la scolarité ultérieure d'un enfant, il semble nécessaire de relativiser ces données quand on aborde le cas des enfants issus de l'immigration (Lorcerie, 1999; Vallet et Caille, 1996). 
Si nous devons retenir quelques enseignements ou conclusions de l'examen du champ de l'expérience scolaire, il est important de souligner au moins deux traits saillants: le premier peut être qualifié de différentialiste, il renvoie à la diversité des positionnements des jeunes en la matière; le second est tendanciel, il renvoie à des dominantes repérées dans ce champ d'expérience. Qu'il s'agisse du vécu relationnel avec les enseignants ou du déroulement concret de la scolarité, qu'il s'agisse du sens donné au fait d'aller à l'école ou de l'appréciation de ses retombées personnelles et professionnelles, des différences apparaissent entre au moins deux groupes de jeunes : ceux qui vivent l'expérience scolaire comme un espace-temps structurant et épanouissant d'un côté, et ceux qui la vivent sur un mode plus difficile, empli d'un sentiment d'incompétence scolaire, de rejet ou d'indifférence, un tempsespace de malentendus et de souffrances de l'autre. Le cas du noyau de jeunes oscillant, selon les items, entre 6 et $15 \%$, qui apprécie négativement ses résultats scolaires tout comme ses rapports aux enseignants et semble exprimer un non-sens de l'investissement scolaire, illustre cette réalité. Le deuxième trait saillant réside dans une tendance globale qui contraste avec l'image répandue quant à la scolarité des jeunes issus de l'immigration maghrébine, souvent présentée sous les signes de l'échec scolaire et d'une crispation des liens avec l'institution scolaire. Révisée à l'aune des résultats obtenus dans cette enquête, une telle image relève davantage de l'imaginaire que de la réalité. La très grande majorité des jeunes vit positivement son expérience scolaire et l'apprécie comme étant structurante personnellement et professionnellement. Cependant, au-delà de cette vue d'ensemble qu'offre l'analyse des items pris isolément, existe-t-il des corrélations entre ces différentes facettes de l'expérience scolaire?

\subsection{De l'unité de l'expérience scolaire?}

L'étude systématique des corrélations entre les différentes variables constitutives de notre champ d'études nous conduit à retenir trois items particulièrement instructifs quant à l'appréhension de la dynamique scolaire dans son ensemble. Il s'agit du niveau scolaire atteint selon qu'il relève du secondaire ou du supérieur, du sens donné à la fréquentation scolaire au lycée et enfin du sentiment de discrimination éventuelle dans l'institution scolaire durant cette même période. Les liens statistiquement significatifs avec certains éléments biographiques ont été également retenus. Notre démarche a consisté en un examen comparatif des effectifs réels et des effectifs théoriques des tableaux relatifs à chaque croisement visant à matérialiser et à localiser les écarts. Toutefois pour réaliser une économie sur le plan de la présentation, nous 
donnerons ici une information synthétique du croisement opéré, de la valeur du Khi2 et du seuil de signification. Quant au sens des variations, il sera donné dans le corps du texte.

\subsubsection{Niveau d'étude et variables associées}

Tableau 1

Niveau d'étude et variables associées

\begin{tabular}{lccc}
\hline $\begin{array}{l}\text { Niveau d'étude croisé avec } \\
\text { des items relatifs à l'expérience scolaire }\end{array}$ & $\begin{array}{c}\text { Valeur } \\
\mathrm{du} \chi^{2}\end{array}$ & $\begin{array}{c}\text { Degré } \\
\text { de } \\
\text { liberté }\end{array}$ & $\begin{array}{c}\text { Seuil de } \\
\text { signification }\end{array}$ \\
\hline Sentiment de compétence à l'école primaire & 18,65 & 1 & 0,001 \\
Sentiment de compétence au lycée & 14,14 & 1 & 0,001 \\
Sens de la scolarité à l'école primaire & 4,44 & 1 & 0,050 \\
Sens de la scolarité au lycée & 13,75 & 1 & 0,001 \\
Appréciation globale des résultats scolaires & 6,98 & 2 & 0,050 \\
\hline
\end{tabular}

Le groupe ayant atteint le niveau supérieur se distingue par rapport à celui du niveau du secondaire par une appréciation positive de ses résultats scolaires $\left(\chi^{2}=6,98, p<0,05\right)$, un sentiment d'être bon élève aussi bien à l'école primaire $\left(\chi^{2}=18,65, \mathrm{p}<0,001\right)$ qu'au lycée $\left(\chi^{2}=14,14, \mathrm{p}<0,001\right)$. Le sens de la scolarité est exprimé davantage en termes d'intérêt et de plaisir qu'en termes d'obligation, de contrainte ou d'habitude, tant au niveau primaire $\left(\chi^{2}=4,44, \mathrm{p}<0,05\right)$ qu'au niveau lycée $\left(\chi^{2}=13,75, \mathrm{p}<0,001\right)$. L'engagement dans les études longues paraît en ce sens étroitement lié à une double dimension : d'une part, une certaine positivité de l'estime de soi scolaire et un sentiment de plaisir et d'intérêt à investir à l'école, d'autre part. 
4.4.2 Sens de la scolarité au lycée et variables associées

Tableau 2

Sens de la scolarité au lycée et variables associées

\begin{tabular}{lccc}
\hline $\begin{array}{l}\text { Sens de la scolarité au lycée croisé avec autres } \\
\text { items biographiques et scolaires }\end{array}$ & $\begin{array}{c}\text { Valeur } \\
\mathrm{du} \chi^{2}\end{array}$ & $\begin{array}{c}\text { Degré } \\
\text { de } \\
\text { liberté }\end{array}$ & $\begin{array}{c}\text { Seuil de } \\
\text { signification }\end{array}$ \\
\hline Sexe & 7,72 & 1 & 0,010 \\
Niveau de scolarité du père & 12,20 & 3 & 0,010 \\
Appréciation globale des résultats scolaires & 4,07 & 1 & 0,050 \\
Sentiment de (non) discrimination au lycée & 4,01 & 1 & 0,050 \\
Rapports avec les enseignants au lycée & 16,77 & 2 & 0,001 \\
Intérêt professionnel de l'école & 13,49 & 2 & 0,005 \\
\hline
\end{tabular}

Le sens donné à la scolarité durant le lycée (16-18 ans) s'avère un indicateur majeur corrélé à quelques caractéristiques individuelles et parentales (sexe du sujet et scolarité du père). Il est également en rapport avec la manière dont s'est déroulée toute une scolarité, tant du point de vue des résultats scolaires que du point de vue du ressenti relationnel. Précisons ici que $94 \%$ de sujets ont atteint le niveau lycée et qu'une hétérogénéité d'orientations caractérise ces lycéens.

Dans le domaine scolaire, le groupe ayant fréquenté le lycée avec plaisir et intérêt se distingue positivement de celui qui s'y rendait par contrainte ou habitude. Le premier enregistre un excédent d'effectifs réels pour souligner des résultats scolaires supérieurs à la moyenne $\left(\chi^{2}=4,07, \mathrm{p}<0,05\right)$, des rapports aux enseignants au lycée plutôt sympathiques $\left(\chi^{2}=16,77, \mathrm{p}<0,001\right)$, une absence ou une rareté d'un ressenti de discrimination $\left(\chi^{2}=4,01\right.$, $\mathrm{p}<0,05)$ ainsi qu'une grande satisfaction quant à l'intérêt professionnel de l'école $\left(\chi^{2}=13,49, \mathrm{p}<0,005\right)$. Le second groupe présente des caractéristiques inverses, c'est-à-dire des résultats scolaires juste à la moyenne, un ressenti de discrimination assez fréquent, des rapports aux enseignants vécus sur les registres de l'antipathie ou de l'indifférence et une insatisfaction par rapport à ce que pourrait apporter l'école professionnellement. Enfin, deux 
particularités distinguent les deux groupes : le niveau scolaire du père et le sexe du sujet. Les pères des jeunes ayant éprouvé intérêt et plaisir au lycée paraissent relativement mieux scolarisés que les pères des jeunes ayant éprouvé contrainte et obligation ou l'ayant fréquenté par habitude $\left(\chi^{2}=12,20, p<0,01\right)$. Les effectifs des pères jamais scolarisés ou ayant le niveau coranique sont excédentaires dans ce dernier cas. En contraste avec les observations récurrentes mettant l'accent sur l'influence du niveau d'instruction de la mère sur la réussite scolaire des enfants (Place et Vincent, 2009), nos résultats pourraient s'expliquer par le niveau scolaire relativement moins faible des pères par rapport aux mères dans cette génération d'immigrés magrébins d'une part, et par la place centrale des pères dans le contexte culturel de l'étude, d'autre part (Gervais, de Montigny, Azaroual et Courtois, 2009, p.29). Enfin, la variable sexe parait déterminante : les filles ont davantage suivi la scolarité avec plaisir et intérêt $\left(\chi^{2}=7,72, p<0,01\right)$ que les garçons. Cette mobilisation scolaire supérieure des filles est attestée dans des travaux menés dans d'autres pays d'accueil tels que la Belgique (De Villers, 2003) ou le Canada (Durand, 2014). Cependant, l'effet de la variable sexe mérite à notre avis d'être étudié en rapport avec l'orientation culturelle des parents (Qribi, 1997) et une articulation avec le sens de la dynamique identitaire spécifique des intéressées (Martinez et Costalat-Founeau, 2005).

\subsubsection{Sentiment de (non) discrimination au lycée et variables associées}

Tableau 3

Sentiment de (non) discrimination au lycée et variables associées

\begin{tabular}{lccc}
\hline $\begin{array}{l}\text { Sentiment de (non) discrimination au lycée } \\
\text { croisé avec le sexe et autres items relatifs à } \\
\text { l'expérience scolaire }\end{array}$ & $\begin{array}{c}\text { Valeur } \\
\mathrm{du} \chi^{2}\end{array}$ & $\begin{array}{c}\text { Degré } \\
\text { de } \\
\text { liberté }\end{array}$ & $\begin{array}{c}\text { Seuil de } \\
\text { signification }\end{array}$ \\
\hline Sexe & 11,49 & 1 & 0,001 \\
Rapports avec les enseignants au lycée & 19,40 & 2 & 0,001 \\
Intérêt de l'école sur le plan professionnel & 13,14 & 2 & 0,005 \\
Intérêt de l'école sur le plan personnel & 6,80 & 2 & 0,010 \\
Sentiment de discrimination au collège & 22,54 & 1 & 0,001 \\
\hline
\end{tabular}

Le ressenti de discrimination raciale au lycée est en corrélation avec les sentiments éprouvés au collège, et ce, dans les mêmes termes $\left(\chi^{2}=22,54, p<0,001\right)$. Quand un tel 
ressenti est exprimé, les rapports avec les enseignants sont vécus davantage sur le registre de l'antipathie, alors que la non-discrimination est corrélée à des rapports vécus plutôt comme agréables et sympathiques $\left(\chi^{2}=19,40, \mathrm{p}<0,001\right)$. De même, l'expérience de discrimination semble impliquer un sentiment d'insatisfaction par rapport à l'école et dans ce qu'elle peut apporter, tant sur le plan personnel $\left(\chi^{2}=6,80, p<0,01\right)$ que professionnel $\left(\chi^{2}=13,14\right.$, $\mathrm{p}<0,05$ ), alors que l'absence de sentiment de discrimination favorise des appréciations plus favorables. Les jeunes de sexe masculin paraissent plus concernés par le phénomène de discrimination que les jeunes de sexe féminin $\left(\chi^{2}=11,49, \mathrm{p}<0,001\right)$. Pourquoi une telle différence ? Certains travaux de recherche évoquent un préjugé positif jouant en faveur des filles par rapport aux garçons. Les représentations dominantes n'épargnent pas les acteurs de l'éducation: les descendantes d'immigrés bénéficieraient d'une meilleure image dans les médias que les garçons (Meurs et Pailhé, 2008) et seraient davantage identifiées par leur sexe que par leur origine ethnique-nationale (Boukhobza, 2005).

\section{Discussion}

Deux faits majeurs ressortent de cette enquête. Le premier réside dans la diversité notoire des expériences scolaires des jeunes issus de l'immigration maghrébine. Ce fait est établi lors de l'examen de la répartition des observations sur les différentes modalités des variables, puis analysé plus finement lors du croisement de ces données. L'hypothèse de la diversité intracatégorielle est en ce sens tout à fait confirmée. Le deuxième fait concerne le système de corrélations significatives entre les dimensions constitutives de l'expérience scolaire des jeunes issus de l'immigration maghrébine. Ainsi avons-nous mis au jour des liens instructifs entre variables définissant l'orientation globale d'une scolarité vécue sous le signe de la réalisation personnelle ou celui de l'impuissance et de l'échec. Il est apparu alors que le sens donné à la fréquentation de l'école, le sentiment de valeur personnelle dans l'exercice du travail d'élève, les rapports affectifs aux enseignants, le ressenti éventuel de discrimination raciale, sont autant d'aspects intimement liés, formant le tissu d'une expérience fondamentale qui influence l'identité d'élève. Le cas des élèves engagés dans les études supérieures est emblématique à cet égard. Ces résultats confirment donc la seconde hypothèse de l'enquête.

Resituée dans le cadre théorique qui a inspiré le questionnement et les dimensions d'investigation, cette enquête permet la mise en relief de formes distinctes d'expériences scolaires dans un milieu socioethnique commun. Les résultats obtenus attestent de la diversité mise en relief dans les travaux de Charlot, Bautier et Rochex (1992), Lahire (1995) et 
Lorcerie (2004). En intégrant les particularités de la condition minoritaire maghrébine et son effet ethnique, cette recherche dépasse l'idée d'une équivalence entre la différence ethnoculturelle et celle de classe sociale soutenue par tout un courant de sociologie critique de l'éducation. En mettant en valeur la variable discrimination comme une composante de l'éprouvé scolaire, les résultats rejoignent les thèses développées par Payet (2000) et Felouzis, Liot et Perroton (2005). Considérée dans sa dimension subjective, l'expérience scolaire révèle des manières distinctes de vivre sa condition d'élève. Dans ce contexte, bien des faits précis, chargés de significations, pourraient nourrir la réflexion sociopédagogique. Il y a d'abord ce cas de figure d'un groupe d'élèves, garçons notamment, qui peine à se construire une identité positive en milieu scolaire et qui semblent cumuler échecs et insatisfactions multiples. Un tel cas illustre la situation des nouveaux lycéens vivant leur expérience sous le signe de l'aliénation tels qu'ils sont identifiés et dépeints par Dubet et Martuccelli (1996). Cette catégorie a retenu l'attention des praticiens et des chercheurs dans la mesure où son mal-être finit par s'exprimer sur des modes qui troublent l'ordre des classes et des établissements (Debarbieux, 1998). Sa distance et ses résistances par rapport aux normes scolaires s'apparentent à une stratégie de contre-acculturation (Périer, 2004). Cependant, il convient de souligner avec force que ce n'est pas là une figure dominante de l'expérience scolaire, mais uniquement une modalité parmi d'autres. La configuration particulière des élèves de sexe féminin retient également l'attention : d'une part, elles éprouvent plus de plaisir et d'intérêt pour les études; d'autre part, elles semblent connaître moins de discrimination. Attestée dans d'autres contextes migratoires, la première particularité pourrait être rapprochée de l'hypothèse psychosociale développée par Martinez et Costalat-Founeau (2005) selon laquelle les élèves maghrébines, comparées à leurs homologues françaises, seraient engagées dans une réussite scolaire confrontée, visant une reconnaissance sociale et une volonté de compensation de certaines victimisations (racisme, par exemple). Les élèves françaises seraient, quant à elles, dans une réussite scolaire normalisée, participant à une construction identitaire qui laisserait davantage de place aux préoccupations de l'adolescence. Cependant, les résultats de notre étude nous invitent à nuancer la portée des victimisations se rapportant à la discrimination raciale. Les filles maghrébines en sont plus épargnées, comparativement aux garçons maghrébins, dans la mesure où, elles seraient, peut-être, plus identifiées au regard de leur sexe que de leur origine ethnique (Boukhobza, 2005). La construction sociale de l'expérience selon la combinaison genre et ethnie en milieu maghrébin représente, en ce sens, un chapitre singulier de la problématique de la diversité scolaire et identitaire intracatégorielle. Plus globalement, évaluée à l'aune des résultats obtenus, la focalisation sur 
l'échec scolaire et les difficultés du système d'éducation à les gérer méritent d'être interrogées. Les rapports multiples à l'institution scolaire et au savoir constituent à cet égard un axe central. Aux yeux de la majorité des sujets de l'enquête, l'école jouit d'une considération positive au regard de ses fonctions et apports. Une telle attitude constitue, selon les travaux portant sur le sens de l'expérience scolaire, une base fondamentale pour l'engagement dans les études. Mais ce rapport à l'institution scolaire se trouve complexifié lorsqu'on introduit la notion de plaisir et d'intérêt, ainsi que celle de la positivité de l'estime de soi scolaire. Ces deux déterminants paraissent associés à la poursuite des études longues. Ce constat souligne la pertinence de la distinction entre une mobilisation sur l'école en tant qu'institution jugée importante et une mobilisation à l'école et en classe, c'est-à-dire dans des activités d'apprentissage et de transformation de soi (Bautier, Charlot et Rochex, 2000; Charlot, 1997). Les dynamiques de personnalisation (Rochex, 2013; Tap, 1988) et la mobilisation forte des familles et des jeunes autour de la réussite à l'école méritent, en conséquence, d'être mieux et plus étudiées. Autre exemple ou fait marquant: les rapports entre éprouvé relationnel avec les cadres pédagogiques d'une part, et investissement et résultats scolaires de l'élève, d'autre part. Le lien entre ces deux variables en contexte scolaire populaire a été confirmé par d'autres recherches mettant l'accent sur la compétence relationnelle comme complément indispensable à la compétence disciplinaire (Beaud, 2002), ou encore l'observation d'une forte demande, chez les élèves, de la prise en compte de leurs singularités et de l'exigence du respect à leur égard (Charlot, 2001). Enfin, dernière observation et non des moindres : compte tenu de la diversité des expériences scolaires mise en relief tant dans cette étude que dans bien d'autres recherches évoquées tout le long de ce développement, ne conviendrait-il pas, chaque fois qu'il est question de scolarité de jeunes issus de l'immigration postcoloniale, de rappeler que cette dernière catégorisation n'est qu'un mot, et de demander : mais de quel jeune précis souhaiterions-nous parler?

\section{Conclusion}

L'analyse statistique des composantes identitaires et subjectives de l'expérience scolaire des jeunes issus de l'immigration maghrébine en France a permis de dégager certaines régularités et de mettre en relief des corrélations significatives entre des facettes de ladite expérience. Les précautions méthodologiques relatives à la formation d'un échantillon homogène, du point de vue de sa composition socioethnique notamment, ainsi que l'examen des variables au moyen d'un tri à plat d'abord, et du $\chi^{2}$ ensuite, ont permis d'établir une certaine diversité intracatégorielle et d'appréhender des cohérences inter-dimensionnelles. L'objectif de l'enquête 
est en ce sens atteint. En effet, loin des représentations communes, généralement négatives, l'étude du public retenu permet l'esquisse d'un paysage nuancé dans lequel les parcours scolaires semblent inséparables des dynamiques subjectives renvoyant au sens de l'investissement scolaire et de l'éprouvé relationnel.

Cependant, des approfondissements demeurent possibles. Dans cette optique, il convient de rappeler que nous avons volontairement centré l'étude sur une région particulière parmi les divers milieux de développement du jeune, région cernée isolément, dans le but d'en étudier le fonctionnement et les articulations internes. Il convient, pour une meilleure intelligibilité du phénomène, de le resituer dans ses rapports à bien d'autres variables sociologiques, biographiques et idiosyncrasiques. La mise en relief du facteur sexe et du facteur niveau scolaire du père donne une indication sur l'intérêt de l'approfondissement de la recherche dans des perspectives intégrant les dimensions biopsychosociales des jeunes et l'approche des effets de l'interstructuration des milieux de vie sur leur développement identitaire (Qribi, Courtinat et Prêteur, 2010). Des approches qualitatives basées sur les histoires de vie, les dires des sujets sur leur parcours et le sens qu'ils donnent aux événements, représentent une voie pertinente pour l'appréhension des processus de socialisation et de personnalisation (Malrieu, 2003), ainsi que le rôle spécifique de l'expérience scolaire et ses transformations dans une telle dynamique (Qribi, 2011).

Sur le plan pédagogique, l'approche de l'expérience scolaire en termes de perception, de sens subjectif, d'horizons, d'attentes..., demeure un axe essentiel pour quiconque souhaite comprendre la construction du sentiment d'aliénation ou de réalisation personnelle. Dans cette perspective, bien des phénomènes qui préoccupent les acteurs de l'éducation, et concernent les conditions de la réussite scolaire, le décrochage, la violence et autres faits dits d'inadaptation scolaire, trouveraient un éclairage instructif si l'on tenait compte de telles dimensions. Cela favoriserait, sans aucun doute, la construction d'expériences susceptible, de permettre aux jeunes de s'approprier les propositions socialisatrices et d'être auteurs de leur présent et de leur avenir.

Monsieur Abdelhak Qribi est Maître de conférences en sciences de l'éducation à l'Université de Guyane. 


\section{ENGLISH TITLE - The educational experience of young people with North African backgrounds in France. Ethnicity and intra-categorical diversity}

SUMMARY - This study analyzes subjective and identity-related aspects of the educational experience of young people with a North African background in France. A survey carried out among 120 men and women, aged 18 to 25, from working-class backgrounds led us to the testing of the intra-categorical diversity hypothesis. Statistical analysis using particularly the $\mathrm{Chi}^{2}$ test highlights significant correlations between different constituent variables of experiences such as alienation or personal fulfillment.

KEY WORDS - Educational experience, North African immigration, intra-categorical diversity, ethnicity

TíTULO - La experiencia escolar de jóvenes provenientes de la inmigración magrebí en Francia. Etnicidad y diversidad intra-categoría

RESUMEN - El estudio presentado se propone examinar algunos aspectos subjetivos e identitarios de la experiencia escolar de jóvenes provenientes de la inmigración magrebí en Francia. Una encuesta por cuestionario realizada con 120 jóvenes de ambos sexos, entre 18 y 25 años, originarios de un medio popular, permite evaluar la hipótesis de la diversidad intracategoría. El análisis estadístico, principalmente usando la chi cuadrado, conduce a resaltar correlaciones significativas entre diferentes variables constitutivas de entramados de experiencias vividas bajo el signo de la alienación o de la realización personal.

Palabras Clave - Experiencia escolar, inmigración magrebí, diversidad intra-categoría, etnicidad, construcción identitaria.

\section{Références}

Abdallah-Pretceille, M. (1986). Vers une pédagogie interculturelle. Paris, France: Institut national de recherche pédagogique.

Bautier, E., Charlot, B. et Rochex, J.-Y. (2000). Entre apprentissage et métier d'élève : le rapport au savoir. Dans A.Van Zanten (dir.), L'école, l'état des savoirs. (p.179-188). Paris, France : La Découverte.

Beaud, S. (2002). $80 \%$ au bac... et après? Paris, France : La Découverte.

Ben Ayed, C. (2011). Discriminations : l'éducation, un espace à haut risque ? Le Sociographe, $34,65-78$.

Benguigui, Y. (2006). Le plafond de verre. [Film documentaire]. [s.1.] : Distribution Ciné Classique.

Berque, J. (1985). L'immigration à l'école de la république. Paris, France : La Documentation française.

Bizet, J.-A. (1985). Les luttes de classes dans l'appareil scolaire et le pluralisme culturel.

Dans C. Clanet (dir.), L'interculturel en éducation et en sciences humaines (p. 145-151). 
Toulouse, France : Presses Universitaires du Mirail (PUM).

Boukhobza, N. (2005). Les filles naissent après les garçons. Représentations sociales des populations d'origine maghrébine en France. Revue européenne des migrations internationales, 21(1), 227-242.

Boulot, S. et Boyzon-Fradet, D. (1988). L'école française : égalité des chances et logiques d'une institution. Revue européenne des migrations internationales, 4(1-2), 49-82.

Brinbaum, Y. (2005). D’une génération à l'autre, les aspirations des familles immigrées : ambition et persévérance. Éducation et formations, 72, 53-75.

Caille, J.-P. (2007). Perception du système éducatif et projets d'avenir des enfants d'immigrés. Éducation et formations, 74, 117-142.

Charlot, B. (1997). Du rapport au savoir. Paris, France : Économica.

Charlot, B. (2001). Le rapport au savoir en milieu populaire. Paris, France : Anthropos.

Charlot, B., Bautier, E. et Rochex, J.-Y. (1992). École et savoir dans les banlieues et ailleurs. Paris, France : Armand Colin.

Chauveau, G. et Rogovas-Chauveau, E. (1990). La (non) réussite scolaire des immigrés; où sont les différences ? Migrants-formation, 82, 25-33.

Cortesero, R. (2010). Au-delà du déclin de l'institution. Éducation et sociétés, 25, 7-16.

Courtinat, A., De Léonardis, M. (2010). Contextes de scolarisation, expérience scolaire et estime de soi chez des collégien (ne)s à haut potentiel. Enfance, 1(10), 99-109.

Debarbieux, E. (1998). Violence et ethnicité dans l'école française. Revue européenne des migrations internationales, 14(1), 77-92.

De Léonardis, M., Féchant, H. et Prêteur, Y. (2005). Modalités de l'expérience scolaire et socialisation familiale chez des collégiens de troisième générale. Revue française de pédagogie, 551,47-59.

Delhomme, P. et Meyer, T. (2002). La recherche en psychologie sociale. Paris, France: Armand Colin.

De Villers, J. (2003). La reproduction impossible. Entreprendre des études universitaires lorsqu'on est descendante d'immigrés maghrébins en Belgique. Éducation et sociétés, 11, 111-123.

Dubet, F. et Martuccelli, D. (1996). À l'école. Sociologie de l'expérience scolaire. Paris, France : Seuil.

Durand, C. (2014). Expérience scolaire des adolescents de la guerre à Montréal : Rapport au savoir et expérience scolaire d'adolescente(s) victimes de la guerre dans une école secondaire à Montréal. Sarrebruck, Allemagne : Presses académiques francophones. 
Felouzis, G., Liot, F., Perroton, J. (2005). L'apartheid scolaire. Enquête sur la ségrégation ethnique au collège. Paris, France : Seuil.

Feyfant, A. (2011). Les effets de l'éducation familiale sur la réussite scolaire. Veille et analyses, 63, 1-14.

Frandji, D. et Rochex, Y. (2011). De la lutte contre les inégalités à l'adaptation aux besoins spécifiques. Éducation et formations, 80, 95-108.

Gervais, C., De Montigny, F., Azaroual, S. et Courtois, A. (2009). La paternité en contexte migratoire : étude comparative de l'expérience d'engagement paternel et de la construction de l'identité paternelle d'immigrants magrébins de première et de deuxième génération. Enfances, familles, générations, 11, 25-43.

Glasman, D. (2001). L'accompagnement scolaire. Sociologie d'une marge de l'école. Paris, France : Presses universitaires de France.

Glaude, M. et Borrel, C. (2002). Les immigrés et leurs descendants sur le marché du travail : un regard statistique. Dans F. Héran (dir.), Immigration, marché du travail, intégration (p. 105-119). Paris, France : Commissariat général du Plan.

Héran, F. (2002). Les recherches sur l'immigration et l'insertion: avancées, débats, perspectives. Dans F. Héran (dir.), Immigration, marché du travail, intégration (p. 11102). Paris, France : Commissariat général du Plan.

Lahire, B. (1995). Tableaux de famille. Heurs et malheurs scolaires en milieux populaires. Paris, France : Gallimard-Le Seuil.

Lescarret, O. (2000). Socialisation familiale, estime de soi, et réussite scolaire (Habilitation à diriger la recherche non publié). Université de Toulouse le Mirail, Toulouse.

Lignon, Y., Rigal, F. et Thiong Ly, A. (2000). Méthodes statistiques fondamentales. Lonrai, France : Édition Ellipses.

Lorcerie, F. (1999). La scolarisation des enfants de migrants : fausses questions et vrais problèmes. Dans P. Dewitte (dir.), Immigration et intégration (p. 212-221). Paris, France : Éditions La Découverte.

Lorcerie, F. (2004). École et appartenance ethnique. Que dit la recherche? Rapport pour le P.I.R.E.F. (Programme incitatif pour la recherche en éducation et en formation). Paris, France : ministère de l'Éducation nationale.

Malrieu, P. (2003). La construction du sens dans les dires autobiographiques. RamonvilleSaint-Agne, France : Éditions ÉRÈS.

Marangé, J. et Lebon, A. (1982). L'insertion des jeunes d'origine étrangère dans la société française. Paris, France : La Documentation française. 
Martinez, N. et Costalat-Founeau, A.-M. (2005). Comparaisons de deux situations de réussite confrontée et normalisée chez des lycéennes d'origine culturelle différente. Connexion, 83 , 149-168.

Merle, P. (2005). L'élève humilié. L'école, un espace de non droit? Paris, France: Presses universitaires de France.

Meurs, D. et Pailhé, A. (2008). Descendantes d'immigrés en France: une double vulnérabilité ? Travail, genre et sociétés, 20, 87-107.

Payet, J.-P. et Van Zanten, A. (1996a). L'école et la question de l'immigration. Revue française de pédagogie, 117, 5-6.

Payet, J.-P. et Van Zanten, A. (1996b). L'école, les enfants de l'immigration et des minorités ethniques. Revue française de pédagogie, 117, 87-149.

Payet, J.-P. (2000). Violence à l'école et ethnicité. Les «raisons pratiques » d'un amalgame. Ville-École-Intégration, enjeux, 121, 190-200.

Périer, P. (2004). Adolescences populaires et socialisations scolaires. Les épreuves relationnelles et identitaires $\mathrm{du}$ rapport pédagogique. Orientation scolaire et professionnelle, (32)2, 227-248.

Perroton, J. (1998). Racisme et école. Orientation scolaire et professionnelle, (numéro spécial : L'expérience scolaire) 2, 271-288.

Perroton, J. (2000). Les dimensions ethniques de l'expérience scolaire. L'année sociologique, $50,437-438$.

Place, D. et Vincent, B. (2009). L’influence des caractéristiques sociodémographiques sur les diplômes et les compétences. Économie et statistiques, 424-425, 125-147.

Potvin, M., Carignan, N., Audet, G., Bilodeau, A. et Deshaies, S. (2010). L'expérience scolaire et sociale des jeunes d'origine immigrante dans trois écoles de milieux pluriethniques et défavorisés de Montréal. Rapport de recherche. Montréal, Québec : Centre de santé et de services sociaux-CSSS et centre affilié universitaire-CAU de BordeauxCartierville-Saint-Laurent.

Qribi, A. (1997). Acculturation et éducation familiale chez les maghrébins dans le contexte français de l'immigration. Bulletin de psychologie, 50(428), 237-242.

Qribi, A., Courtinat, A. et Prêteur, Y. (2010). Socialisation interculturelle et dynamique identitaire chez les jeunes adultes issus de l'immigration maghrébine en France. International review of education, 56(5-6), 683-703.

Qribi, A. (2011). De la chrysalide à l'envol : transmission identitaire et personnalisation. Le cas de deux jeunes femmes issues de l'immigration maghrébine. Dans O. Coulon- Reveyrand 
et Z. Guerraoui (dir.), Transmission familiale et interculturelle : ruptures, aménagements, créations (p. 107-126). Paris, France : In Press.

Qribi, A. (2012). Socialisation interculturelle et identité. Paris, France : L'Harmattan.

Rochex, J-Y. (2013). Expérience scolaire et subjectivation. Dans A. Baubion-Broye, R. Dupuy, Y. Prêteur (dir.) : Penser la socialisation en psychologie. Actualité de l'œuvre de Philippe Malrieu (p. 85-97). Toulouse, France : Éditions ÉRÈS.

Tap, P. (1988). La société Pygmalion. Intégration sociale et réalisation de la personne. Paris, France : Dunod Éditeur.

Vallet, L.-A. et Caille, J.-P. (1996). Les élèves étrangers ou issus de l'immigration dans l'école et le collège français. Une étude d'ensemble. Les dossiers d'Éducation et formations, 67, Paris, France : ministère de l'Éducation nationale.

Zephir, S. (2007). Différentes modalités de l'expérience minoritaire dans l'espace urbain d'une zone d'éducation prioritaire: les effets paradoxaux d'une action positive (Thèse de doctorat non publiée). Université de Nice, Nice.

Zimmermann, D. (1982). La sélection non verbale à l'école, Paris, France : ESF éditeur.

\section{Correspondance}

abdelhak.qribi@espe-guyane.fr

\section{Contribution de l'auteur}

Abdelhak Qribi: $100 \%$

Ce texte a été révisé par Viviane Boucher

Texte reçu le : 21 septembre 2013

Version finale reçue le : 2 novembre 2015

Accepté le : 14 janvier 2016 